\title{
A conserved MRF4 promoter drives transgenic expression in Xenopus embryonic somites and adult muscle
}

\author{
TIMOTHY J. HINTERBERGER* \\ Biomedical Program, University of Alaska Anchorage, Anchorage, AK, USA
}

\begin{abstract}
The muscle regulatory factor MRF4 is expressed in both embryonic and adult vertebrate skeletal muscle cells. In mammals the MRF4 gene has a complex cis-regulatory structure, with many kilobases $(\mathrm{kb})$ of upstream sequence required for embryonic expression in transgenic mice. Here, initial functional comparison between Xenopus and mammalian MRF4 genes revealed that 610 base pairs (bp) of the XMRF4a proximal promoter drove substantial transgenic expression in $X$. laevis myogenic cells, from somites of neurula embryos through adult myofibers, and as little as 180 bp gave detectable expression. Over 300 bp of XMRF4a proximal promoter sequence is highly conserved among three $X$. laevis and $X$. tropicalis MRF4 genes, but only about 150 bp shows significant identity to mammalian MRF4 genes. This most-conserved XMRF4a region contains a putative MEF2 binding site essential for expression both in transgenic embryos and in transfected mouse muscle cells. A rat MRF4 minimal promoter including the conserved region also was active in transgenic $X$. laevis embryos, demonstrating a striking difference between the mouse and Xenopus transgenic systems. The longest XMRF4a promoter construct tested, with 9.5 kb of 5 '-flanking sequence, produced significantly greater expression in transfected mouse cells than did promoters $4.3-\mathrm{kb}$ or shorter, suggesting that the intervening region contains an enhancer, although no increased expression was evident when this region was included in transgenic $X$. laevis embryos. Further identification and analysis of Xenopus MRF4 transcriptional control elements will offer insights into the evolution of this gene and of the myogenic gene regulatory network.
\end{abstract}

KEY WORDS: Xenopus, transgenic, muscle, MRF4, promoter

\section{Introduction}

Within the genetic and epigenetic regulatory network that governs skeletal myogenesis in mammals, one transcription factor family, comprising MRF4, MyoD, myogenin and Myf5, occupies a central position (Pownall et al., 2002; Berkes and Tapscott, 2005; Bryson-Richardson and Currie, 2008). These four closely related myogenic regulatory factors (MRFs) control gene expression during myoblast specification and myofiber differentiation, maintenance, hypertrophy, repair, and regeneration, but the MRFs' individual roles in these cellular events remain incompletely understood. Though MRF4 was for some years regarded primarily as a myofiber differentiation factor and unimportant for myoblast determination, which was thought to depend instead on MyoD and Myf5, more precise gene targeting showed that MRF4can function as a determination gene when MyoD and Myf5 are absent (Kassar-Duchossoy et al. 2004). The revised model, in which either Myf5 or MRF4 acts upstream of MyoD to direct embryonic multipotent mesodermal cells into the myogenic lineage, is more consistent with the observations that MRF4 transcripts appear before those of $M y O D$ and precede or are contemporaneous with those of Myf5 in both mouse somites (Summerbell et al., 2002) and chick limbs (Lin-Jones and Hauschka, 1996).

In vitro and transgenic approaches have both contributed to our current understanding of mammalian MRF4regulation. Transfection of cells in vitro showed that E-box motifs (CANNTG) in rat and mouse MRF4 proximal promoters are required for their activation by MyoD, myogenin, or Myf5. MEF2 binding, at a site encompassing the TATA box, is also required for maximal muscle-

Abbreviations used in this paper: GFP, green fluorescent protein; MRF, myogenic regulatory factor.

\footnotetext{
*Address correspondence to: Dr. Timothy J. Hinterberger. Biomedical Program, University of Alaska Anchorage, 3211 Providence Drive, Anchorage, AK 99508, USA. Fax: +1-907-786-4700. e-mail: timh@uaa.alaska.edu
} 
specific expression (Black et al., 1995; Naidu et al., 1995). Although 130 base pairs (bp) of rat MRF4 proximal promoter suffices for expression in transfected muscle cells, greater expression is seen with 5 kilobases $(\mathrm{kb})$ of upstream sequence (Hinterberger et al., 1992), which includes an enhancer region comprising several E-boxes and a consensus MEF2 site (Kerkvliet and Hinterberger, 1997). The proximal promoter by itself has no activity in myotomes and very little in myofibers of transgenic mice, but $8.5 \mathrm{~kb}$ of 5 '-flanking sequence activates expression in thoracic somitic myocytes and in fetal myofibers (Pin et al., 1997). When added to the proximal promoter, the enhancer at $-5 \mathrm{~kb}$ drives increases expression chiefly in fast muscle fibers at fetal stages but fails to activate expression in the embryonic somites (Pin and Konieczny, 2002). Similarly for the mouse MRF4 gene, a 7.5-kb promoter fragment drives partial expression in somites (Fomin etal. 2004), but fuller expression depends on an enhancer located between $-88 \mathrm{~kb}$ and $-140 \mathrm{~kb}$ from the MRF4 coding region (Carvajal et al., 2001). Recent work (Carvajal et al., 2008) shows that numerous enhancers 5 ' of the MRF4 coding region interact in mutually exclusive ways with the MRF4 promoter and the closely linked Myf5 promoter in mouse. One particular enhancer located at $-8 \mathrm{~kb}$ from the MRF4 coding region was previously shown to direct temporally and spatially distinct activity from the two promoters (Chang et al., 2004, 2007).

A

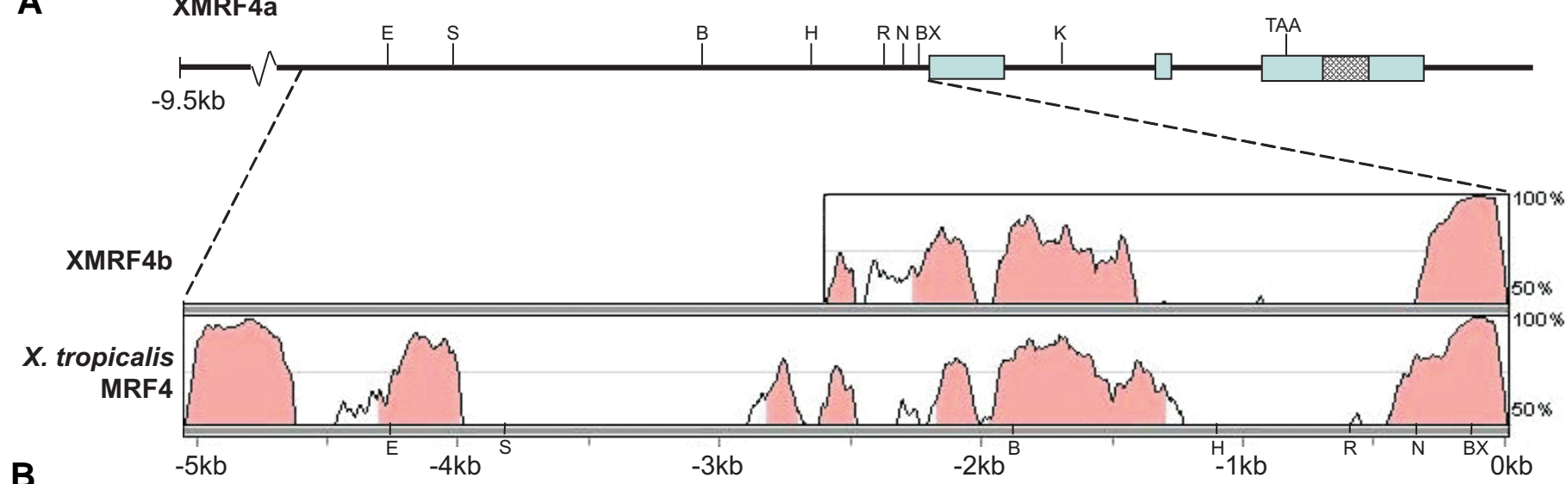

B

$\downarrow-610$

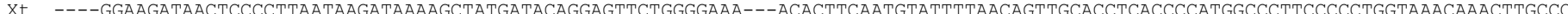
Xa GAATTCTGGCAAAAACATTTCAATCTGAGAAAAACCATATAGAAT

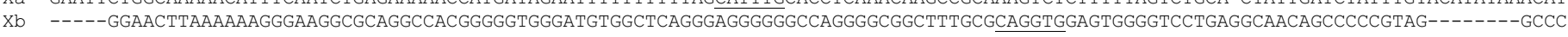
* $* * * *$ * $*$ *

GAATTCAGTTGAACTCTGGAGAgGGGGGAGGAGCAGGGCAGTGTTAATCC

TGAAATCCTTTTTGTGGCAGC-TCCAACTTGCATGTAGCATTTTTGTTCCTTTACATTATAAATAATATGCTTATTATTAACAAATACTT--TAGTATTGTTAATTAATAGAAATAACTGAATACTGAAA AAGGGTGC---ATATGCTTAC-ACTGGGGTGCATACA-CATTTGTGTTCCTTTATGTTAAAAATAATATTCATAATATTATTAACTAATA--CAGTGAGGTATATTAATAGGAATAACTGAAT-------
CGGACCCCCAGTCCGACACCGAATAAAGCAATAGGCTGGTTCGCTTCCAATAAAGATTAATTATATCTTAGTTTTGATCAAGTACACGGTACTGTTCTATTTTTACAGAGAAAAGGGAAATCATTTT

\section{$\downarrow-360$}

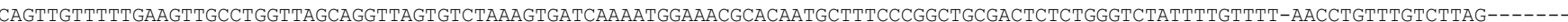
ACTCCATGGCATGTTTA------GGGGCCAGATAATTAAATCTTGCATTAAAAGTGCATCTTTGTTGCACAAATATGGCAGCTTATTATTCATATAAATGTA----AACTAGTAGACATTTTATTATGT ACCCCATGGCATGGTTA------GGGGTAAGATCATTCATTCTTGCATTAGAAGTGCATCTTTGTTGCACACATAGGGCTTCTTTTTATTTATTTAAATGTCTAATAATTAGTAGACATGTTATTATTT TTTTTAAAAATGGATTATTTGGATGAAATGGAGTCTTCCATTATTGCCTTAAAAATGTATCTTTTTTGCACAAATAGGGCTTCTTATTATTTATATAAATGTCTATTAGTAGGTAGATATTTTATTATGT

$$
\text { - *** * }
$$

$\bullet \bullet * * \star * * * \bullet * \bullet \bullet * \bullet * * \bullet * \bullet \bullet * * * * * * * * * * * * * *$

Ra GGACTGTCATATTCACTCTGAGAGGTGACATTTCTCCGATTTCTTTCAAACTAGA-TGTTCTGGGGAGCACTA

XT TTTGGTTGCTGAGTCTTGTCCAAAGGGATCTGATTCCATACAGGAGATGATGAGAGCTGCATCCAGGAGZ

$\mathrm{Xa}$ TTTTTTTGCTGATTCGTGTCCAAAGGGATCTGGTTCCATTCAGGAGATGATGAGGGCTGCACCCAGGAGA

Xb tTTGATTGCTGATTCTTGTCCAAAGGGATCTGGTTCCATACAGGAGATGATGAGGGCTGTACCCAGGAG

$\downarrow-120$

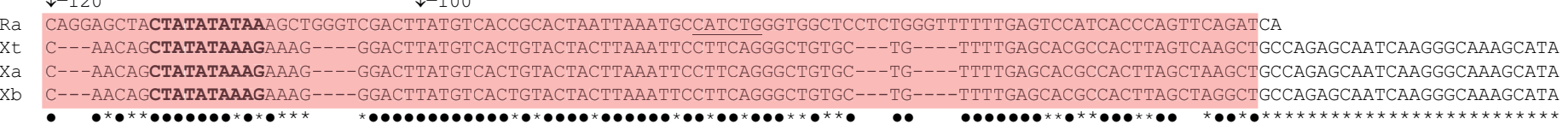

Fig. 1. Structure of the XMRF4a genomic clone and comparison to other MRF4 gene sequences. (A) Upper portion of the figure shows the $5^{\prime}-$ flanking region, exons, introns, and 3'-flanking sequence of the 13-kb clone. Restriction enzyme sites mentioned in the text (B, Bam $H$ l; $B X$, Bst $X$ I; E, Eco R V; H, Hind III; K, Kpn I; N, Nco I; R, Eco R I; S, Sac I) and the stop codon are indicated. Cross-hatching identifies the P2X7 gene homology region (see text). Lower portion shows results of VISTA alignments of 5066 bp of XMRF4a 5'-flanking sequence versus XMRF4b and X. tropicalis MRF4 loci. Shading indicates conservation of $70 \%$ or greater. For XMRF4b, only 2325 bp of sequence was available for comparison. (B) Alignment of nucleotide sequences from rat MRF4 (Ra), X. tropicalis MRF4 (Xt), XMRF4a (Xa), and XMRF4b (Xb) promoters. Numbering refers to positions 5 ' from the XIMRF4a start codon. -610 indicates the Eco R I site, -360 the Nco / site, -180 the Bst $X$ I site, and -120 and -100 indicate the $5^{\prime}$ ends of PCR-generated deletions. The MEF2 site is in bold type and E boxes (potential MRF binding sites) are underlined. Black dots beneath nucleotides indicate conservation in all four species, while asterisks indicate conservation only among the threeXenopus genes. Shading covers the 150-bp highly conserved region described in the text. 
The MRF family is conserved throughout the vertebrate classes, yet the genes' individual expression patterns differ among representatives of the classes that have been examined. Several differences between rodents and Xenopus exemplify this. In mice, myogenin is expressed in the myotomes, while in Xenopus, Xmyogenin mRNA reportedly appears only in the secondary myogenesis of limbs and dorsal body muscles during metamorphosis (Nicolas etal., 1998). In rats (Hinterberger etal., 1991) and in mice (Miner and Wold, 1990), MRF4 mRNA is more abundant than MyoD mRNA in adult muscle, whereas in Xenopus the reverse holds true (Jennings, 1992). Muscle denervation in rats leads to increased MRF4 transcript levels (Adams et al., 1995), but denervation of Xenopus muscle leads to decreased levels of XMRF4 mRNA (Jennings, 1992; Nicholas et al., 2000). These observations indicate that the MRF gene regulatory network must function somewhat differently in Xenopus than it does in mammals.

Here, I show that Xenopus myogenic cells require only a short MRF4promoter for transgenic activity. Approximately $180 \mathrm{bp} 5$ ' to the start codon of an $X$. laevis MRF4 gene sufficed for transgenic expression in embryonic myotomes. A rat MRF4 minimal promoter, containing a core sequence that is conserved in all vertebrate MRF4genes, also drove expression in transgenic Xenopus embryos, clearly demonstrating a major functional difference between the mouse and frog transgenic assay systems. Postmetamorphic transgenic animals bearing either the rat or the Xenopus minimal promoter displayed reporter expression in trunk, limb and cranial muscles. Including additional sequence up to $610 \mathrm{bp} 5^{\prime}$ of the start codon resulted in greater embryonic expression. Although transgenesis assays gave no evidence of any cis-regulatory activity located 5 ' of the 610-bp promoter, sequence comparison among Xenopus MRF4 genes showed strong conservation of several upstream regions, and transient transfection of mouse $\mathrm{C} 2 \mathrm{C} 12$ myoblasts pointed to an enhancer between $4.3 \mathrm{~kb}$ and $9.5 \mathrm{~kb} 5$ ' of the coding region.

\section{Results}

\section{Gene cloning and sequence analysis}

Two distinct $X M R F 4$ sequences, apparently corresponding to the two MRF4 loci in the duplicated genome of $X$. laevis, were represented in multiple clones from a lambda genomic library. One clone of XMRF4a (gene designation consistent with Della Gaspera et al., 2006) contained the full coding sequence in three exons, approximately $9.5 \mathrm{~kb}$ of 5 '-flanking sequence, and approximately 1 kb of 3'-flanking sequence (Fig. 1A). An XMRF4b (Della Gaspera et al., 2006) clone with all three exons was partially sequenced but the lengths of its flanking regions were not determined. The two XMRF4 genes displayed greater than 93\% identity for approximately $330 \mathrm{bp} 5$ ' to the start codon. This region included a TATA box that is conserved in all available vertebrate MRF4 gene sequences. The mammalian, lizard and chicken MRF4 genes also contain a MEF2 binding site (CTATATATAA) that overlaps the TATA box; in $X$. laevis and $X$. tropicalis MRF4 genes, the corresponding site (CTATATAAAG) deviated at one nucleotide from a MEF2 consensus site [YTA $\left(A / T_{4}\right)$ TAR (Black and Olson, 1998)]. A 150-bp region flanking the TATA box and putative MEF2 site in the Xenopus MRF4 genes displayed $71 \%$ identity with the corresponding region of the rat MRF4 promoter (Fig. 1B). No E boxes were present within the conserved proximal 330-bp region of any of the three Xenopus MRF4 genes. In $X M R F 4 a$, three $E$ boxes lie at $-450 \mathrm{bp},-475 \mathrm{bp}$ and $-555 \mathrm{bp}$ from the first codon, and one is found in a similar location in $X M R F 4 b$ and in $X$. tropcalis MRF4.

Another region of conservation between $X M R F 4 a$ and $-b$ began in XMRF4aapproximately $1.1 \mathrm{~kb}$ upstream of the end of the 330-bp proximal region and extended at least $1.2 \mathrm{~kb}$ upstream from there. It was conserved between $X M R F 4 a$ and $X$. tropicalis MRF4 as well. VISTA alignment with the $X$. tropicalis sequence indicated the existence of an even more strongly conserved region beginning about $4 \mathrm{~kb}$ upstream from the XMRF4a start codon and continuing to the end of the available 9673-bp XMRF4a sequence (Fig. 1A). No identity with the Xenopus MRF4 conserved upstream regions was indicated in any other available vertebrate genome sequence by VISTA or BLAST analysis.

A BLASTN search of GenBank using the XMRF4a sequence as a query showed that the $3^{\prime}$ untranslated region of XMRF4a contains a segment nearly identical to a portion of the coding sequence of an unrelated gene in $X$. laevis (Fig. 1A). A 289-bp sequence region, flanked by TA repeats, displayed $96 \%$ identity to a fragment of the cDNA for the P2X7 ATP-gated ion channel (Paukert et al., 2002).

\section{Endogenous MRF4 expression in X. laevis}

In situ hybridizations showed XMRF4 transcripts in the presomitic mesoderm beginning by at least stage 15 (Fig. 2A), consistent with our previous RT-PCR results (Ataian et al., 2003). Expression continued in somites and myotomes at all stages

Fig. 2. Whole mount in situ hybridization of $X$. laevis embryos with the full-length XMRF4 probe or XMyf5 probe. All are oriented anterior to the right. (A) Stage 1415 early neurula, dorsal view. XMRF4 expression is seen in presomitic mesoderm prior to segmentation, as well as in the anterior. (B) Stage 20 neurula, lateral view. XMRF4 staining is most intense in the somites but also evident in the eye primordia. (C) Stage 16 neurula, dorsolateral view. $\mathrm{XMyf5}$ expression is confined to the posterior mesoderm. (D) Stage 31-32 tailbud embryo, lateral view. In addition to the myotomal staining, XMRF4 expression is evident in the eyes, brain, branchial arches, otic vesicles, and head mesoderm. (E) Stage 31-32 tailbud embryo, lateral view. XMyf5 expression is seen in tailbud mesoderm, dorsal and ventral myotomal cells, and in primordia of some cranial muscles.

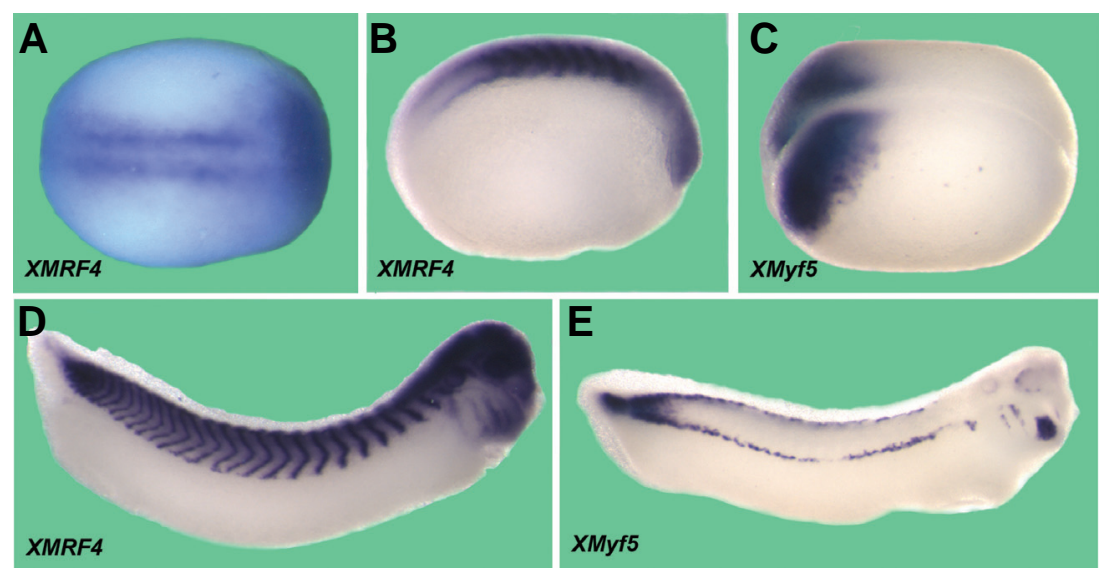




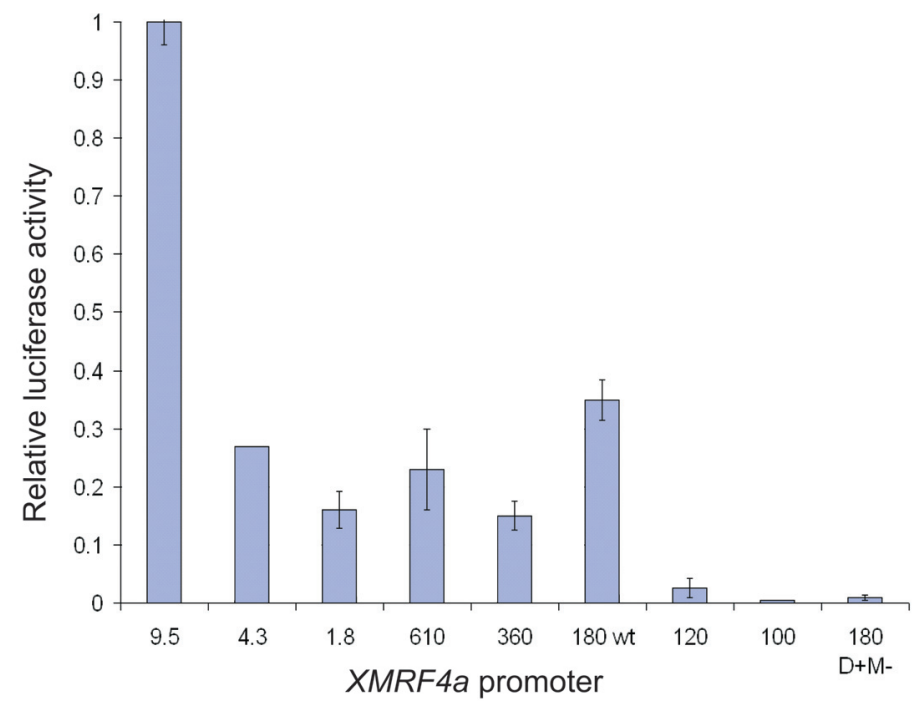

Fig. 3. Relative activity of XMRF4a promoters in mouse myotubes. The graph shows averages of values \pm s.e.m. from two to four independent transient transfection experiments for each XMRF4a construct, except for 4.3-kb which is a single datum. Each experimental value is the average of two separate $\mathrm{C} 2 \mathrm{C} 12$ cell transfections, expressed as the ratio of test construct to RL-SV4O control, normalized to the value obtained for a positive control construct.
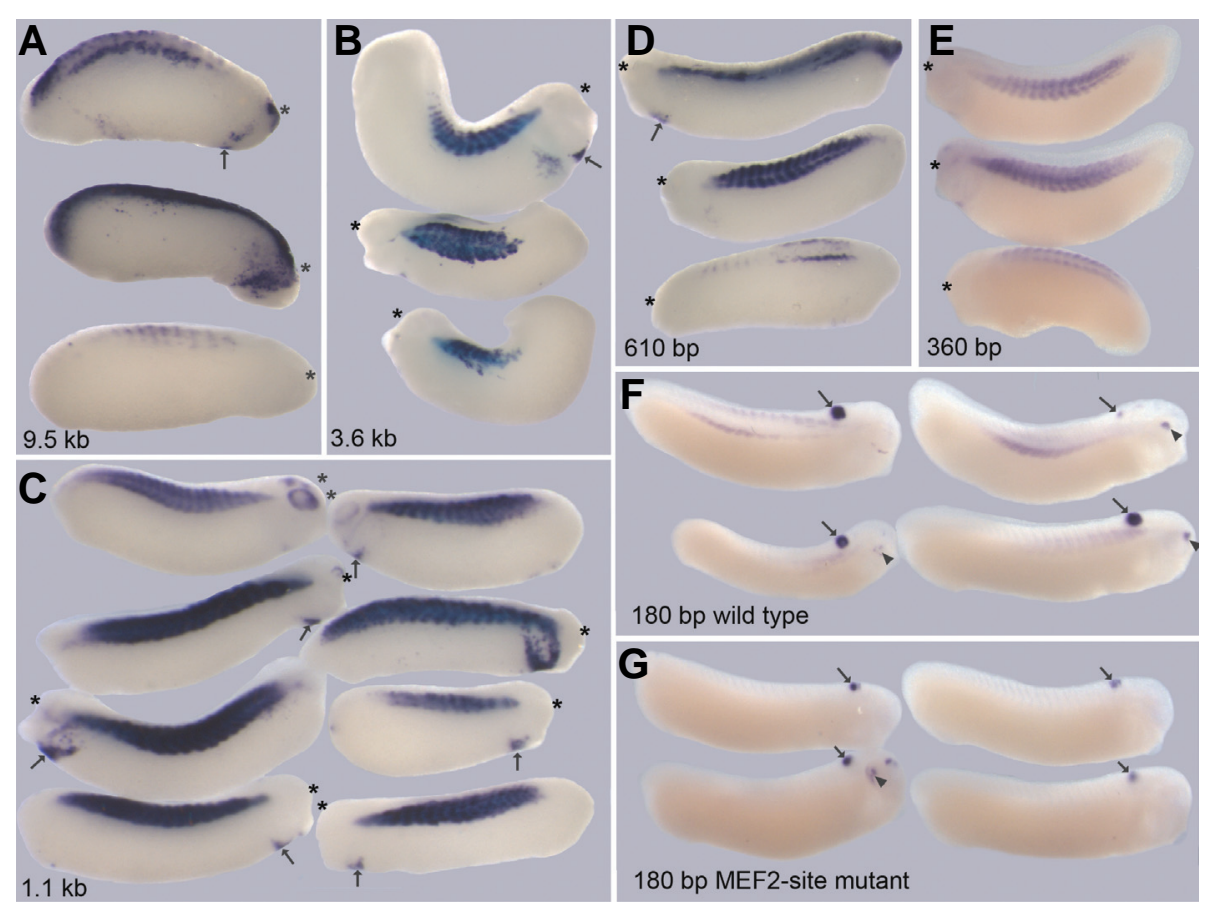

180 bp wild type

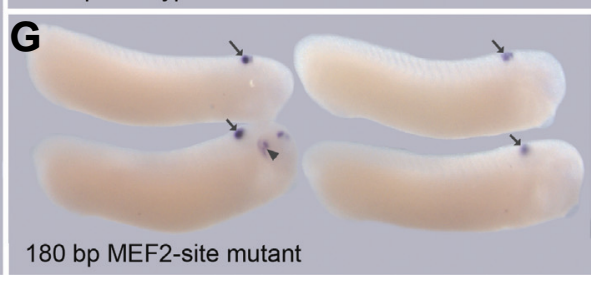

Fig. 4. Relative activity of XMRF4a promoters in transgenic $X$. laevis embryos. Lengths of the promoters (described in the text) are indicated. Each panel shows lateral views of representative positive individuals from a single experiment. All specimens are oriented with dorsal sides towards the top of the figure, but because expression often was asymmetric, either the left or right side may be shown, whichever had more intense staining, and the anterior end is marked with an asterisk. In panels (A-D), arrows indicate ectopic expression in cardiogenic regions. Embryos in $(\mathbf{F}, \mathbf{G})$ are co-transgenic for both the indicated XMRF4a-GFP construct and the gamma-crystallin-GFP construct; all are oriented with the anterior to the right side of the figure. Staining of the hindbrain (arrows) and, in some cases, the lens of the eye (arrowheads), results from gamma-crystallin-GFP expression. examined (Fig. 2 B,D) with transcripts confined to the perinuclear region of myocytes. Unexpectedly, staining was also seen in the structures (Fig. 2B,D); this expression will be described more fully sewhere. XMyf5expression pattern in conjunction with that of XMRF4. As demonstrated by other investigators (e.g., Martin and Harland, ; Polli and Amaya, 2002), XMyf5transcripts were detected in myotomes. XMyf5 expression was also seen in branchial arches and in primordia of cranial and extraocular muscles of late tailbud ryos; however, no XMyf5expression was detected in neural structures, and at every stage its pattern was clearly distinct from

\section{Cell transfections}

Transient transfection of $\mathrm{C} 2 \mathrm{C} 12$ mouse myoblasts with XMRF4a 5 '-flanking sequence fragments (hereafter called "promoters" even if several $\mathrm{kb}$ in length) linked to a luciferase reporter demonstrated that the TATA/MEF2 site plus approximately $60 \mathrm{bp}$ of additional 5 ' sequence (180wt) constitutes a minimal promoter in myotubes that differentiated from these cells (Fig. 3). Constructs that included only 6 bp of XMRF4a sequence $5^{\prime}$ to the TATA/ MEF2 site (120) had greatly reduced expression, while constructs in which the promoter was truncated $10 \mathrm{bp}$ downstream of the site (100) showed no activity. When the TATA/ MEF2 site was mutated to abolish MEF2 binding (180 D+M-), promoter activity also was lost. Longer constructs that included genomic sequence as far as $4.3 \mathrm{~kb} 5^{\prime}$ from the first XMRF4acodon resulted in slightly less expression than did the minimal promoter. However, when the entire available $9.5 \mathrm{~kb}$ of XMRF4a 5 'flanking sequence was included, expression occurred at levels approximately threefold greater than that from the minimal promoter, implying the presence of an enhancer located between $-4.3 \mathrm{~kb}$ and $-9.5 \mathrm{~kb}$ that is active in mammalian myotubes.

\section{Transgenic animals}

To study XMRF4a transcriptional regulation in $X$. laevis embryonic cells in vivo, test promoters were linked to a green fluorescent protein (GFP) cDNA and inserted as transgenes. Tailbud embryos were examined rather than earlier stages in order to allow for accumulation of detectable transcript levels from low-activity insertion sites. Constructs containing $9.5 \mathrm{~kb}, 3.6 \mathrm{~kb}, 1.1 \mathrm{~kb}, 610 \mathrm{bp}, 360$ $\mathrm{bp}$, or $180 \mathrm{bp}$ of wild-type XMRF4apromoter sequence all were expressed in myotomal muscle (Fig. $4 \mathrm{~A}-\mathrm{F}$ ), at frequencies ranging 
TABLE 1

FREQUENCY OF TRANSGENIC MYOTOMAL EXPRESSION BY MRF4 PROMOTERS

\begin{tabular}{|c|c|c|}
\hline Promoter & Experiment no. & GFP+ embryos \\
\hline \multicolumn{3}{|c|}{ XMRF4a promoters } \\
\hline $9.5 \mathrm{~kb}$ & $\begin{array}{l}1 \\
2 \\
\text { total }\end{array}$ & $\begin{array}{l}24 \%(11 / 46) \\
22 \%(15 / 69) \\
23 \%(26 / 115)\end{array}$ \\
\hline $3.6 \mathrm{~kb}$ & $\begin{array}{l}1 \\
2 \\
3 \\
\text { total }\end{array}$ & $\begin{array}{l}27 \%(25 / 94) \\
15 \%(8 / 53) \\
59 \%(10 / 17) \\
26 \%(43 / 164)\end{array}$ \\
\hline $1.1 \mathrm{~kb}$ & $\begin{array}{l}1 \\
2 \\
3 \\
\text { total }\end{array}$ & $\begin{array}{l}36 \% 87 / 239 \\
8 \%(10 / 126) \\
55 \%(40 / 73) \\
31 \%(137 / 438)\end{array}$ \\
\hline 610 bp & $\begin{array}{l}1 \\
2 \\
3 \\
\text { total }\end{array}$ & $\begin{array}{l}67 \%(8 / 12) \\
7 \%(3 / 44) \\
57 \%(12 / 21) \\
30 \%(23 / 77)\end{array}$ \\
\hline 360 bp & $\begin{array}{l}1 \\
2 \\
\text { total }\end{array}$ & $\begin{array}{l}20 \%(26 / 129) \\
25 \%(1 / 4) \\
20 \%(27 / 133)\end{array}$ \\
\hline 180 bp (wt) & $\begin{array}{l}1 \\
2 \\
3 \\
\text { total }\end{array}$ & $\begin{array}{l}8 \%(13 / 158) \\
6 \%(9 / 147) \\
20 \%(5 / 25) \\
8 \%(27 / 330)\end{array}$ \\
\hline 180 bp (D+M-) & $\begin{array}{l}1 \\
2 \\
\text { total }\end{array}$ & $\begin{array}{l}0 \%(0 / 120) \\
0 \%\left(1^{\star} / 228\right) \\
0 \%\left(1^{*} / 348\right)\end{array}$ \\
\hline \multicolumn{3}{|c|}{ rat MRF4 promoter } \\
\hline 410 bp & $\begin{array}{l}1 \\
2 \\
\text { total }\end{array}$ & $\begin{array}{l}7 \%(6 / 87) \\
12 \%(15 / 123) \\
10 \%(21 / 210)\end{array}$ \\
\hline
\end{tabular}

*faint, questionable staining

from $8 \%$ to $31 \%$ (Table 1). In repeated experiments with the same construct, I obtained large variations in the percentage of positive embryos, preventing statistically valid quantitative ranking among these promoters based on their expression frequencies alone. When the gamma-crystallin-GFP construct was included as a control, successful transgene insertion could be inferred from GFP expression in the hindbrain and (at late tailbud stages) in the lenses of the eyes. Among these reporter-positive embryos, the 3.6-kb and 1.1-kb XMRF4apromoters gave myotomal expression in large majorities of cases, whereas the 180 -bp promoter gave expression in less than a third of cases (Table 2). The intensity of in situhybridization staining in the myotomes varied greatly within all groups of embryos carrying the same XMRF4aGFP construct. However, the intensity of staining seen with the 360 -bp or 180-bp promoters never reached the maximal level seen with the longer promoters. There was also a tendency for expression from the two shorter promoters to be confined to the dorsal and ventral ends of myotomes (Fig. $4 \mathrm{E}, \mathrm{F}$ ). (Unlike the endogenous XMRF4staining, GFP reporter transcripts were not localized to the perinuclear region of myocytes, possibly because they lack a 3' untranslated region.) One other qualitative difference was that the 610-bp and longer promoters occasionally produced expression in the branchial arches, in the cardiogenic region and the vicinity of eyes (Fig. 4 A-D), a result not seen with the two shorter promoters. Transgenic embryos in which GFP was under the control of a 2.3-kb $X M R F 4 b$ promoter showed myotomal expression identical to that seen with similar-sized XMRF4a constructs (data not shown).

Just as with mouse myoblasts transfected in vitro, mutation of the MEF2 site in the 180-bp XMRF4a promoter essentially abolished reporter gene expression in myotomal muscle of transgenic
TABLE 2

\section{FREQUENCY OF CO-EXPRESSION OF $\gamma$-CRYSTALLIN AND XMRF4A PROMOTERS}

\begin{tabular}{ll} 
XMRF4a promoter length & \% crys-GFP+ that were also XMRF4a-GFP+ $^{+}$ \\
\hline $3.6 \mathrm{~kb}$ & $88 \%(14 / 16)$ \\
$1.1 \mathrm{~kb}$ & $93 \%(67 / 72)$ \\
$180 \mathrm{bp}$ (wild-type) & $30 \%(13 / 43)$ \\
$180 \mathrm{bp}(\mathrm{D}+\mathrm{M}-)$ & $2 \%(1 * / 60)$ \\
\hline
\end{tabular}

embryos. In one such experiment, inclusion of the gammacrystallin-GFP control resulted in expression in brain or lens in $26 \%$ of the specimens, confirming that transgene insertion had taken place in those embryos despite the absence of myotomal expression (Fig. 4G).

To test the hypothesis that a minimal mammalian MRF4 promoter, due to its strong identity with the Xenopus proximal promoters, would be active in $X$. laevis embryos, 410 bp of rat MRF4 promoter sequence (Hinterberger et al., 1992; Naidu et al., 1995) was linked to the GFP cDNA. The rat promoter produced expression similar to that seen with the shorter XMRF4apromoters both in frequency (Table 1) and in pattern and intensity (Fig. 5).

Transgenic tadpoles carrying the XMRF4a-GFP constructs were raised through metamorphosis in order to determine whether their transgenes would be expressed in limb muscles. The 3.6-kb, 1.1-kb, 610-bp (Fig. 6A) and 180-bp promoters all drove GFP expression in post-metamorphic skeletal muscles in the trunk, limbs, and head (animals bearing the 360 -bp promoter have not yet been fully examined). In sections of transgenic hindlimb muscle, GFP expression was apparently restricted to myofibers (Fig. 6 B,C).

In order to compare the onset of XMRF4aGFP transgene expression to that of the endogenous XMRF4 genes, stronglyexpressing transgenic adults were mated with wild types to provide embryos for analysis of early stages. F1 offspring carrying the XMRF4a 610-bp promoter construct had detectable GFP expression in somites by at least stage 20 (Fig. 7A). F1 embryos carrying the $3.8-\mathrm{kb}$ and the $1.1-\mathrm{kb}$ transgenes showed similar

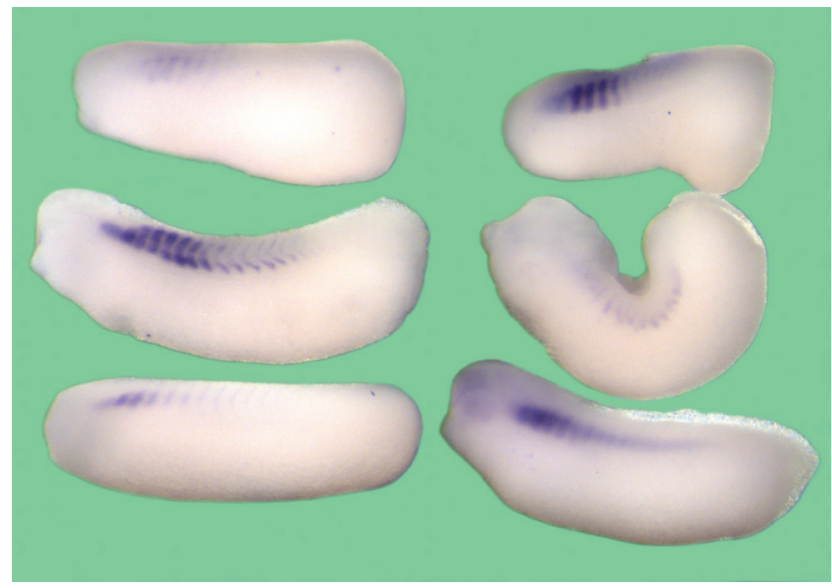

Fig. 5. Activity of the rat MRF4 minimal promoter in transgenic $X$. laevis embryos. In situ hybridization shows GFP expression in representative positive individuals, oriented with dorsal side towards the top of the figure, anterior towards the left. 

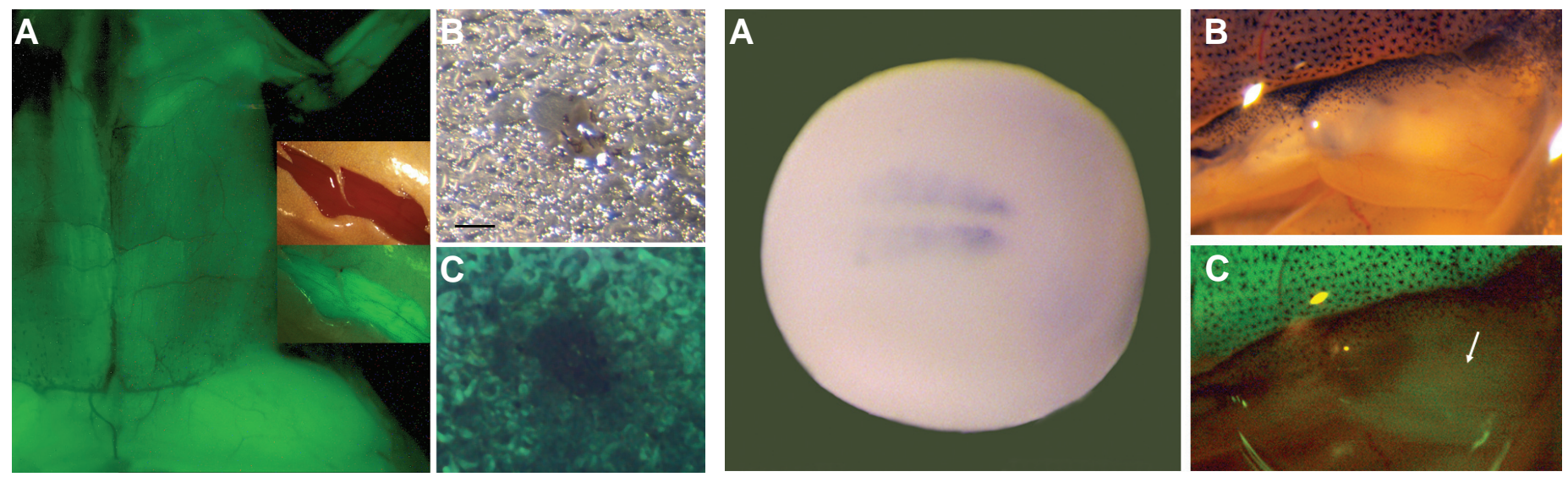

Fig. 6 (Left). Postmetamorphic expression of XMRF4a transgenes. (A) GFP fluorescence driven by the 610-bp promoter in skeletal muscle, ventral view, anterior at top, skin removed. This is a composite of two separate exposures of the same animal. Most of the trunk and the left forelimb and proximal hindlimb are shown, but the head is out of the frame. Inset shows reflected-light (upper) and fluorescence (lower) images of a ventral skin incision in the hindlimb of a frog carrying the 1.1-kb promoter. GFP fluorescence in muscle is clearly distinguishable by color from autofluorescence in the skin. (B) Reflected-light image of a $50 \mu \mathrm{m}$ frozen section of hindlimb muscle from anXMRF4a-1.1-GFP transgenic frog. Note the neurovascular bundle in the center. (C) GFP fluorescence in the same section as in (B). Scale bar, $100 \mu \mathrm{m}$.

Fig. 7 (Right). Onset of XMRF4a transgene expression. (A) In situ hybridization showing GFP expression under control of the 610-bp promoter in the early somites of an F1 individual at stage 18-20, dorsal view, anterior to the right. (B) Reflected-light image of the right hindlimb of a stage-56 F1 animal carrying the 1.1-kb promoter construct. (C) GFP fluorescence from the same limb as in (B). Note that the developing muscle (arrow) in the limb shows much lower intensity of fluorescence than do the tail myotomes at the top of the figure.

results (data not shown). During metamorphosis of these animals, GFP fluorescence became detectable in hindlimb muscle at approximately stage 56 (Fig. 7 B,C).

\section{Discussion}

With this study, Xenopus laevis MRF4 becomes the second member of the $X$. laevis MRF gene family, after $X M y f 5$ (Yang et al., 2002), subjected to transgenic analysis of its cis-regulatory regions. Embryonic myotomes and, later, limb muscles expressed an XMRF4a proximal promoter as short as $-180 \mathrm{bp}$ from the first codon, while a 610-bp promoter produced somewhat stronger embryonic expression.

Promoter expression in any transgenic founder reflects a balance between the inherent cell-type-specific transcriptional activity of the test sequence and the repressive properties of the chromosomal site into which it randomly inserts. In principle, a stronger promoter will show detectable activity even in relatively repressive genomic sites, and in permissive sites it will appear more active than weaker promoters. The longer promoters tested here, ranging from $610 \mathrm{bp}$ up to $9.5 \mathrm{~kb}$, all gave detectable expression in myotomes at similar frequencies, while the 360-bp and 180-bp wild-type promoters displayed lower expression frequencies. Large batch-to-batch variances reduced the statistical significance of these differences when test constructs alone were inserted, but inclusion of the gamma-crystallin-GFP construct as a marker of transgene co-insertion served to filter the data so that much larger differences were seen between the 1.1-kb and 180bp constructs' expression frequencies, consistent with their relative intensities of in situhybridization staining. Taken together, the expression frequencies and the staining intensities suggest that the 610-bp promoter contains the all regulatory elements required for XMRF4a expression in myotomes of tailbud embryos, al- though its initial expression appeared somewhat delayed and more restricted than the endogenous gene (compare Figs. 2A and $B$ with Fig. 7A).

These results stand in striking contrast to those obtained with mouse or rat MRF4 promoters in transgenic mice, where the regulatory activity required for detectable somitic expression is found at least $5.6 \mathrm{~kb}$ upstream from the coding region (Fomin et al., 2004). Yet as shown here, a rat MRF4 410-bp proximal promoter is at least minimally effective in transgenic Xenopus, demonstrating an important functional difference between the two transgenic systems. This conclusion differs from previous studies of mammalian promoters in transgenic $X$. laevis (e.g., Beck and Slack, 1999; Lim et al., 2004) which emphasized the similarities of transgene expression in the two systems. In those studies, however, proximal promoters that lacked activity in mice were not tested in for activity Xenopus.

$X M R F 4 a$ promoter fragments were examined for their efficacy driving reporter expression in mouse muscle cells in vitroas well as in transgenic $X$. laevis. Here too, the two types of data are not congruent. Luciferase assays of transfected mouse myotubes showed that the 9.5-kb promoter was very effective, while the 4.3$\mathrm{kb}$ to 180 -bp promoters were less but about equally effective. This result suggests the existence of an enhancer located between -4.3 $\mathrm{kb}$ and $-9.5 \mathrm{~kb}$ from the coding region. Paradoxically, however, this $X$. laevis sequence region failed to display enhancer activity in transgenic embryos; promoters from $9.5 \mathrm{~kb}$ down to $610 \mathrm{bp}$ in length performed indistinguishably. In addition, the in vitroresults point more strongly to a central role for the -180 promoter than do the transgenics, where this promoter displayed minimal activity.

Within the 180-bp XMRF4apromoter, only the 150 bp flanking the putative MEF2 site has significant sequence identity to mammals, and truncation of the rat $0.4-\mathrm{kb}$ promoter has shown that this conserved region accounts for all of its in vitroactivity (Naidu etal., 
1995). Deletion of the 5 ' end of this region from the XMRF4a-180 promoter to make the -120 promoter essentially eliminated its in vitro activity, suggesting that these approximately $60 \mathrm{bp}$ have a key role both in mammals and in Xenopus. As it is in the mammalian MRF4 (Black et al., 1995, Naidu et al., 1995) and Xenopus MyoDa (Leibham etal., 1994) genes, the putative MEF2 binding site in the XMRF4a minimal promoter was obligatory for expression. Loss of MEF2 binding to the XMRF4a-180D+Mpromoter was not tested biochemically in this study; however, electrophoretic mobility shift assays of an identical mutant MEF2 site in Xenopus MyoDashowed that in vitrosynthesized XMEF2A protein, as well as nuclear extracts from developing embryos or from C2 myotubes, bound to the wild-type but not to the mutant site (Leibham et al., 1994; Wong et al., 1994).

In rat and mouse MRF4 promoters as well as in many other muscle genes, MEF2 acts synergistically with MRF proteins, which bind at E-boxes in the proximal promoter. The three Eboxes in the 610-bp XMRF4a promoter have not been tested functionally, and it is unknown whether they are binding sites for Xenopus MRF proteins, but the lower transgenic activity observed for the 360-bp promoter, which lacks these sites, is consistent with a possible role of the E-boxes in activating transcription.

Sequence alignment of $X$. laevis XMRF4a and XMRF4b and $X$. tropicalis MRF4 genes shows conserved and non-conserved regions within their first few kb of 5'-flanking sequence, but expression differences between XMRF4aconstructs did not coincide with the boundaries of conserved regions. The 1.1-kb promoter lacks the conserved regions found between $-1 \mathrm{~kb}$ and $-3 \mathrm{~kb}$ in the 3.6$\mathrm{kb}$ promoter and upstream of $-4 \mathrm{~kb}$ in the $9.5-\mathrm{kb}$ promoter, yet all three constructs gave very similar patterns and intensities of reporter gene expression. While evolutionary sequence constraint is associated with an observed genome function in many cases (Hardison, 2000), other constrained regions likely have critical functions not detected by experimental assays employed thus far (de la Calle-Mustienes et al., 2005; Margulies et al., 2007). If the Xenopus MRF4 and Myf5 genes have overlapping regulatory regions as they do in mammals (Carvajal et al., 2001, 2008), some of the conserved sequence 5 ' to their coding region will likely contain regulatory elements functionally associated with the Myf5 promoter rather than with MRF4. Whatever their functions, the presence of strikingly similar patterns of sequence conservation between the $X$. laevis MRF4a and $-b$ loci and between $X$. laevis and $X$. tropicalis MRF4, with no similarity to mammals, strongly suggests that the conserved regions exert control over aspects of skeletal muscle development unique to frogs. Conversely, non-conserved as well as conserved sequence elements must contribute to the regulation of XMRF4a and $-b$ because their transcript levels differ during the course of muscle development and during regeneration (Della Gaspera et al., 2006).

The 610-bp and longer XMRF4a promoters showed nonmyotomal transgenic expression in some cases, albeit in a pattern that differed from that of endogenous $X M R F 4$, which showed extrasomitic expression domains in brain, eye, branchial arches, and other anterior structures. Because it followed a consistent pattern in multiple founder embryos, transgene expression in the cardiogenic and branchial regions at tailbud stage may not represent simply random ectopic expression resulting from the transgenesis procedure (i.e., misregulated transcription of randomly inserted or extrachromosomal DNA, or aberrant cell movements due to embryo manipulation). Non-myotomal XMRF4a transgene expression may instead represent an abnormal manifestation, in the experimental context, of normal XMRF4a expression in anterior structures. This context includes the absence of potential intronic and 3 ' regulatory regions and the absence of a linked Myf5 promoter, which in transgenic mice results in abnormal MRF4 promoter activity (Carvajal et al., 2008). Furthermore, sequence upstream of $-9.5 \mathrm{~kb}$ may be required for activating a normal XMRF4a expression pattern in the brain, eyes, and other anterior structures.

This initial study of the cis-regulatory structure of the XMRF4a gene in comparison to mammalian MRF4 has identified a highlyconserved core promoter that sufficed for transgenic myotomal and postmetamorphic expression in $X$. laevis, demonstrated that additional elements within -610 bp of the XMRF4acoding region enhanced myotomal expression; pointed to an upstream enhancer within the -9.5-kb region; and shown that use of different transgenic and in vitro assays can lead to very different results. Identification and analysis of the putative upstream enhancer, as well as finer-scale deletion and point-mutation analysis of the XMRF4a610-bp promoter, to fully understand their activities both in mammalian cells and in transgenic Xenopus embryos, should provide insight into the evolution of transcriptional control of this gene. Establishment of additional breeding lines carrying these transgenic constructs will facilitate discovery of the regulatory elements involved in XMRF4atranscriptional responses to physiological events such as denervation and muscle injury.

\section{Materials and Methods}

\section{Isolation and sequence analysis of XMRF4 promoters}

A 230-bp Not-Pvull fragment at the 5' end of a Xenopus laevis MRF4 cDNA (Jennings, 1992) was used to screen a genomic library (Lambda Fix II, Stratagene). Recovered inserts were subcloned into pBluescript (Stratagene). One insert of about $13 \mathrm{~kb}$ in length, designated XMRF4a, was sequenced through 9673 bp (GenBank Accession No. EU045573) including 5066 bp 5 ' to the start codon. From another insert, designated XMRF4b, a 6-kb EcoR I fragment was partially sequenced including 2325 bp 5 ' to the start codon (GenBank Accession No. EU045574). The XMRF4a 5' sequence was used as a query for a BLAST search of the $X$. tropicalis v4.1 sequence (Joint Genome Institute, http://www.jgi.doe.gov) that identified the orthologous region of the $X$. tropicalis genome (scaffold 5,1883000 to 1888563). The VISTA computational tool [http:// genome.lbl.gov./vista (Brudno et al., 2007)], with the calculation window set to $100 \mathrm{bp}$, was used for comparisons between these 5 ' sequence regions. An alignment of the $X$. tropicalis region against other vertebrate genomes was inspected at the UCSC Bioinformatics Site [http:// genome.ucsc.edu (Kent et al., 2002)].

\section{XMRF4a promoter-reporter gene constructs}

After subcloning a 1.9-kb EcoR I-Kpn I fragment containing $610 \mathrm{bp}$

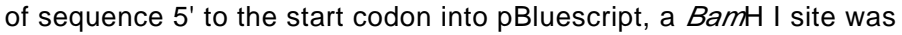
introduced just ahead of the start codon by PCR with proofreading polymerase (Vent, New England Biolabs). This 610-bp EcoR I-BamH I fragment was then used to generate shorter promoters, by restriction digestion at an $\mathrm{Nco}$ I site (-360 bp) or a BstX I site (-180 bp), and by PCR to introduce a Kpn I site about 6 bp upstream of the MEF2/TATA site $(-120 \mathrm{bp})$ or about 6 bp downstream of the MEF2/TATA site $(-100$ bp). PCR-based modifications utilized primers consisting of $18-20 \mathrm{bp}$ of $X M R F 4 a$ sequence with an additional restriction enzyme recognition site. 
A mutated version of the 180-bp promoter designed to prevent binding of MEF2 to its site ( $\mathrm{D}+\mathrm{M}-)$, as previously demonstrated in the Xenopus XMyoDa promoter (Leibham et al., 1994), was made by site-directed mutagenesis (MORPH kit, 5-prime-to-3-prime Inc., Boulder, CO). The mutagenic oligonucleotide (CAACAGgTATATAAgcAAAGGG) differed from the wild-type sequence at the nucleotides shown in lower-case letters. Other promoter constructs were generated from the full-length XMRF4aclone by using PCR with proofreading polymerase (DyNAzyme, Finnzymes Oy., Espoo, Finland) to introduce an Xhol site just ahead of the start codon and a KpnI site at the 5 ' end of the clone. The resulting 9.5-kb product was inserted into a plasmid and subsequently digested with $E_{C O R}$ V, Sacl, BamH I, or with Hind III to create a 4.3-kb, a 3.6-kb, a 1.8-kb, and a 1.1-kb promoter, respectively.

Green fluorescent protein reporter constructs were made by excising the GFP5 (Siemering et al., 1996):SV40 polyadenylation cassette from $X N k \times 2.5-G F P 5$ (Sparrow et al., 2000) with KpnI and Not I and inserting it into pBluescript. XMRF4apromoter fragments were then inserted at the Kpn I site. A mammalian MRF4-GFP5 promoter-reporter construct was produced by inserting the -336 to +71 fragment of rat MRF4 (Naidu et al., 1995). Luciferase reporter constructs for cell transfection were made by ligating the XMRF4a promoter fragments into pGL2-Basic (Promega). Procedural details are available on request. All mutants and constructs were verified by sequencing.

\section{Generation and analysis of transgenic Xenopus laevis}

MRF4-GFP5 andngamma-crystallin-GFP3 (Offield et al., 2000) plasmids were digested with restriction enzymes to release the promoterreporter cassettes, which were then separated from the vector by agarose gel electrophoresis and extracted from the gel with clean-up mini-columns (Qiagen or Promega). For a positive control in some experiments, the gamma-crystallin-GFP3 construct was included along with the XMRF4a-GFP5 construct, as embryos incorporating one construct have a high probability of incorporating another co-injected construct as well (Hartley et al., 2001). Approximately $100 \mathrm{ng}$ of each DNA was used per transgenesis reaction by the method of Smith etal. (2006), which is based on the original REMI method (Kroll and Amaya, 1996) but omits egg extract and restriction enzyme from the pre-injection incubation of DNA with sperm nuclei. GFP mRNA was routinely visualized in embryos fixed at tailbud stage by in situhybridization (Sive et al., 2000) with a digoxigenin-labeled probe transcribed from the first $380 \mathrm{bp}$ of the GFP5 cDNA, with BM Purple (Roche) as the substrate for alkaline phosphatase. In some experiments, living GFP $^{+}$embryos were identified by epifluorescence microscopy (Leica MZFLIII) and raised through metamorphosis. In order to examine transgene expression in early embryos, sexually mature transgenic individuals were mated with wild-type individuals. For visualization of GFP in adult tissues, post-metamorphic animals expressing GFP were anesthetized deeply with benzocaine, skinned, and photographed with epifluorescence. The animals were then sacrificed. Animal care and euthanasia followed protocols approved by University of Alaska Anchorage IACUC.

\section{In situ hybridization probes for endogenous XMRF4 mRNA and for XMyf5 mRNA}

An antisense digoxigenin-labeled RNA probe was transcribed from the full-length XMRF4a cDNA followed by alkaline hydrolysis to produce fragments several hundred bp in length. A probe for $X M y f 5$ was transcribed from a 470-bp EcoR I-Pst fragment of the cDNA (Hopwood et al., 1991). In situ hybridization was performed as for the GFP probe.

\section{Cell culture and transfection}

C2C12 mouse myoblasts were grown in DMEM with $20 \%$ fetal bovine serum (FBS) and were plated at approximately $50 \%$ confluence on gelatin-coated 24-well plates prior to transfection. Equal molar amounts of the different XMRF4a-GL2 plasmids were used, together with a constant amount of renilla luciferase plasmid RL-SV40 (Promega) as a control for transfection efficiency. Following transfection using Qiagen Superfect or GTS GenePorter2 reagent, cells were maintained in $20 \%$ FBS for 6 hours and then switched to 2\% horse serum to promote differentiation for 5 days before harvest and assay (Dual-Luciferase Kit, Promega).

\section{Acknowledgements}

I thank Sylvia Evans and Rob Grainger for providing me with opportunities to learn frog transgenesis in their laboratories and for plasmids. Many of my students at the University of Alaska Anchorage contributed to aspects of this project. This work was supported by grants from NIH (HD35326) and from the Alaska BRIN program.

\section{References}

ADAMS, L., CARLSON, B.M., HENDERSON, L. and GOLDMAN, D. (1995) Adaptation of nicotinic acetylcholine receptor, myogenin, and MRF4 gene expression to long-term muscle denervation. J Cel/ Bio/131: 1341-1349.

ATAIAN, Y., OWENS, J. and HINTERBERGER, T. (2003). MRF4 gene expression in Xenopus embryos and aneural myofibers. Dev Dyn 226: 551-554.

BECK, C.W. and SLACK, J.M. (1999). Gut specific expression using mammalian promoters in transgenic Xenopus laevis. Mech Dev 88: 221-227.

BERKES, C.A. and TAPSCOTT, S.J. (2005). Myod and the transcriptional control of myogenesis. Semin Cell Dev Biol16: 585-595.

BLACK, B.L., MARTIN, J.F. and OLSON, E.N. (1995). The mouse MRF4 promoter is trans-activated directly and indirectly by muscle-specific transcription factors. $J$ Biol Chem 270: 2889-2892.

BLACK, B.L. and OLSON, E.N. (1998). Transcriptional control of muscle development by myocyte enhancer factor-2 (MEF2) proteins. Annu Rev Cell Dev Biol 14: 167-196.

BRUDNO, M., POLIAKOV, A., MINOVITSKY, S., RATNERE, I. and DUBCHAK, I. (2007). Multiple whole genome alignments and novel biomedical applications at the vista portal. Nucleic Acids Res. 35(Web Server issue):W669-674

BRYSON-RICHARDSON, R.J. and CURRIE, P.D. (2008). The genetics of vertebrate myogenesis. Nat Rev Genet 9: 632-646.

CARVAJAL, J.J., COX, D., SUMMERBELL, D. and RIGBY, P.W. (2001). A BAC transgenic analysis of the MRF4/MYF5 locus reveals interdigitated elements that control activation and maintenance of gene expression during muscle development. Development 128: 1857-1868.

CARVAJAL, J.J., KEITH, A. and RIGBY, P.W. (2008). Global transcriptional regulation of the locus encoding the skeletal muscle determination genes MRF4 and MYF5. Genes Dev 22: 265-276.

CHANG, T.H., PRIMIG, M., HADCHOUEL, J., TAJBAKHSH, S., ROCANCOURT, D., FERNANDEZ, A., KAPPLER, R., SCHERTHAN, H. and BUCKINGHAM, M. (2004). An enhancer directs differential expression of the linked MRF4 and MYF5 myogenic regulatory genes in the mouse. Dev Bio/269: 595-608.

CHANG, T.H., VINCENT, S.D., BUCKINGHAM, M.E. and ZAMMIT, P.S. (2007) The a17 enhancer directs expression of MYF5 to muscle satellite cells but MRF4 to myonuclei. Dev Dyn 236: 3419-3426.

DE LA CALLE-MUSTIENES, E., FEIJOO, C.G., MANZANARES, M., TENA, J.J., RODRIGUEZ-SEGUEL, E., LETIZIA, A., ALLENDE, M.L. and GOMEZSKARMETA, J.L. (2005). A functional survey of the enhancer activity of conserved non-coding sequences from vertebrate iroquois cluster gene deserts. Genome Res 15: 1061-1072.

DELLA GASPERA, B., SEQUEIRA, I., CHARBONNIER, F., BECKER, C., SHI, D.L. and CHANOINE, C. (2006). Spatio-temporal expression of MRF4 transcripts and protein during Xenopus laevis embryogenesis. Dev Dyn 235: 524-529.

FOMIN, M., NOMOKONOVA, N. and ARNOLD, H.H. (2004). Identification of a critical control element directing expression of the muscle-specific transcription factor MRF4 in the mouse embryo. Dev Bio/272: 498-509.

HARDISON, R.C. (2000). Conserved noncoding sequences are reliable guides to regulatory elements. Trends Genet 16: 369-372.

HARTLEY, K.O., HARDCASTLE, Z., FRIDAY, R.V., AMAYA, E. and PAPALOPULU, N. (2001). Transgenic Xenopus embryos reveal that anterior neural development requires continued suppression of BMP signaling after gastrulation. Dev 
Bio/238: 168-184.

HINTERBERGER, T.J., MAYS, J.L. and KONIECZNY, S.F. (1992). Structure and myofiber-specific expression of the rat muscle regulatory gene MRF4. Gene 117: 201-207.

HINTERBERGER, T.J., SASSOON, D.A., RHODES, S.J. and KONIECZNY, S.F. (1991). Expression of the muscle regulatory factor MRF4 during somite and skeletal myofiber development. Dev Bio/147: 144-156.

HOPWOOD, N.D., PLUCK, A. and GURDON, J.B. (1991). Xenopus MRF-5 marks early muscle cells and can activate muscle genes ectopically in early embryos. Development 111: 551-560.

JENNINGS, C.G. (1992). Expression of the myogenic gene MRF4 during Xenopus development. Dev Biol 151: 319-332.

KASSAR-DUCHOSSOY, L., GAYRAUD-MOREL, B., GOMES, D., ROCANCOURT, D., BUCKINGHAM, M., SHININ, V. and TAJBAKHSH, S. (2004). Mrf4 determines skeletal muscle identity in myf5:Myod double-mutant mice. Nature 431: 466-71.

KENT, W.J., SUGNET, C.W., FUREY, T.S., ROSKIN, K.M., PRINGLE, T.H., ZAHLER, A.M. and HAUSSLER, D. (2002). The human genome browser at ucsc. Genome Res 12: 996-1006.

KERKVLIET, C.M. and HINTERBERGER, T.J. (1997). Distal regulatory regions of the rat MRF4 gene. Biochem Biophys Res Commun 237: 170-176.

KROLL, K.L. and AMAYA, E. (1996). Transgenic Xenopus embryos from sperm nuclear transplantations reveal FGF signaling requirements during gastrulation. Development 122: 3173-3183.

LEIBHAM, D., WONG, M.W., CHENG, T.C., SCHROEDER, S., WEIL, P.A., OLSON, E.N. and PERRY, M. (1994). Binding of TFIID and MEF2 to the TATA element activates transcription of the Xenopus Myoda promoter. Mol Cel/ Biol 14: 686-699.

LIM, W., NEFF, E.S. and FURLOW, J.D. (2004). The mouse muscle creatine kinase promoter faithfully drives reporter gene expression in transgenic Xenopus laevis. Physiol Genomics 18: 79-86.

LIN-JONES, J. and HAUSCHKA, S.D. (1996). Myogenic determination factor expression in the developing avian limb bud: An RT-PCR analysis. DevBio/174: 407-422.

MARGULIES, E.H., COOPER, G.M., ASIMENOS, G., THOMAS, D.J., DEWEY, C.N., SIEPEL, A., BIRNEY, E., KEEFE, D., SCHWARTZ, A.S., HOU, M. et al. (2007). Analyses of deep mammalian sequence alignments and constraint predictions for $1 \%$ of the human genome. Genome Res 17: 760-774.

MARTIN, B.L. and HARLAND, R.M. (2001). Hypaxial muscle migration during primary myogenesis in Xenopus laevis. Dev Bio/239: 270-280.

NAIDU, P.S., LUDOLPH, D.C., TO, R.Q., HINTERBERGER, T.J. and KONIECZNY, S.F. (1995). Myogenin and MEF2 function synergistically to activate the MRF4 promoter during myogenesis. Mol Cel/ Bio/15: 2707-2718.

NICOLAS, N., GALLIEN, C.L. and CHANOINE, C. (1998). Expression of myogenic regulatory factors during muscle development of Xenopus: Myogenin mRNA accumulation is limited strictly to secondary myogenesis. Dev Dyn 213: 309321.

OFFIELD, M.F., HIRSCH, N. and GRAINGER, R.M. (2000). The development of Xenopus tropicalis transgenic lines and their use in studying lens developmental timing in living embryos. Development 127: 1789-1797.

PAUKERT, M., HIDAYAT, S. and GRUNDER, S. (2002). The P2X(7) receptor from Xenopus laevis. Formation of a large pore in Xenopus oocytes. FEBS Lett513: 253-258

PIN, C.L. and KONIECZNY, S.F. (2002). A fast fiber enhancer exists in the muscle regulatory factor 4 gene promoter. Biochem Biophys Res Commun 299: 7-13.

PIN, C.L., LUDOLPH, D.C., COOPER, S.T., KLOCKE, B.J., MERLIE, J.P. and KONIECZNY, S.F. (1997). Distal regulatory elements control MRF4 gene expression in early and late myogenic cell populations. Dev Dyn208: 299-312.

POLLI, M. and AMAYA, E. (2002). A study of mesoderm patterning through the analysis of the regulation of XMyf-5 expression. Development 129: 2917-2927.

POWNALL, M.E., GUSTAFSSON, M.K. and EMERSON, C.P., JR. (2002). Myogenic regulatory factors and the specification of muscle progenitors in vertebrate embryos. Annu Rev Cell Dev Bio/18: 747-783.

SIEMERING, K.R., GOLBIK, R., SEVER, R. and HASELOFF, J. (1996). Mutations that suppress the thermosensitivity of green fluorescent protein. Curr Bio/ 6: 1653-1663.

SIVE, H.L., GRAINGER, R.M. and HARLAND, R.M. (2000). Early development of Xenopus laevis: A laboratory manual. Cold Spring Harbor Laboratory Press, Cold Spring Harbor, New York.

SMITH, S.J., FAIRCLOUGH, L., LATINKIC, B.V., SPARROW, D.B. and MOHUN, T.J. (2006). Xenopus laevistransgenesis by sperm nuclear injection. Nat Protoc 1: $2195-2203$

SPARROW, D.B., CAI, C., KOTECHA, S., LATINKIC, B., COOPER, B., TOWERS, N., EVANS, S.M. and MOHUN, T.J. (2000). Regulation of the tinman homologues in Xenopus embryos. Dev Bio/227: 65-79.

SUMMERBELL, D., HALAI, C. and RIGBY, P. (2002). Expression of the myogenic regulatory factor MRF4 precedes or is contemporaneous with that of Myf5 in the somitic bud. Mech Dev 117: 331-335.

WONG, M.W., PISEGNA, M., LU, M.F., LEIBHAM, D. and PERRY, M. (1994). Activation of Xenopus myod transcription by members of the MEF2 protein family. Dev Bio/166: 683-695.

YANG, J., MEI, W., OTTO, A., XIAO, L., TAO, Q., GENG, X., RUPP, R.A. and DING, X. (2002). Repression through a distal TCF-3 binding site restricts Xenopus myf5 expression in gastrula mesoderm. Mech Dev115: 79-89. 


\section{Further Related Reading, published previously in the Int. J. Dev. Biol.}

See Special Issue Pattern Formation edited by Michael K. Richardson and Cheng-Ming Chuong at:

http://www.ijdb.ehu.es/web/contents.php?vol=53\&issue=5-6

Myoskeletin, a factor related to Myocardin, is expressed in somites and required for hypaxial muscle formation in Xenopus Hui Zhao, Martha L. Rebbert and Igor B. Dawid

Int. J. Dev. Biol. (2007) 51: 315-320

Limb muscle development.

Bodo Christ and Beate Brand-Saberi

Int. J. Dev. Biol. (2002) 46: 905-914

Involvement of myogenic regulator factors during fusion in the cell line C2C12.

Stéphane Dedieu, Germain Mazères, Patrick Cottin and Jean-Jacques Brustis

Int. J. Dev. Biol. (2002) 46: 235-241

Control of the expression of the Mrf4 and Myf5 genes: a BAC transgenic approach

JJ Carvajal, D Cox, D Summerbell, PWJ Rigby

Int. J. Dev. Biol. (2001) 45: S139-S140

Targeted inactivation of myogenic factor genes reveals their role during mouse myogenesis: a review.

$\mathrm{H} \mathrm{H}$ Arnold and T Braun

Int. J. Dev. Biol. (1996) 40: 345-353

5 yr ISI Impact Factor $(2008)=3.271$
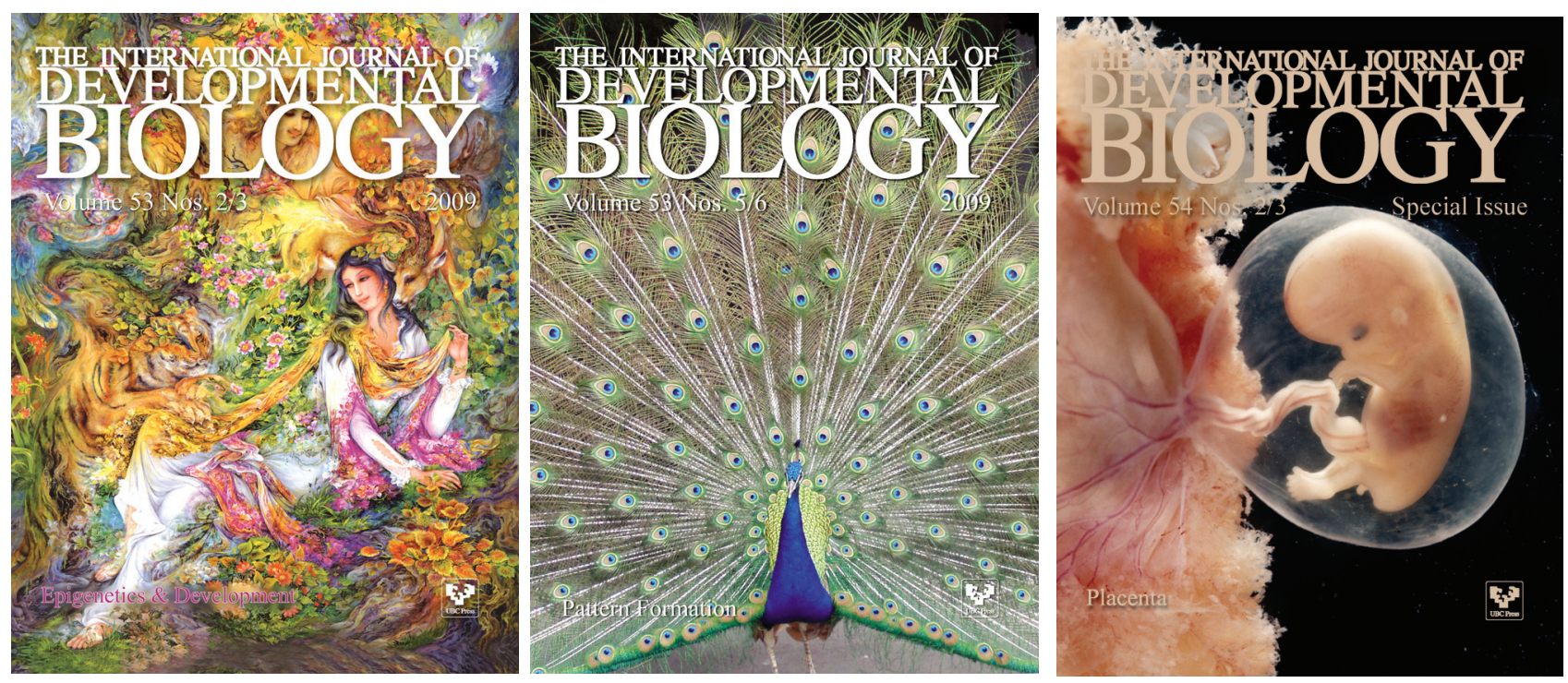